\title{
A living-sphere approach for locally oriented sustainable design
}

\author{
Hideki Kobayashi $^{1}$ (D) Shinichi Fukushige ${ }^{1}$
}

Received: 22 February 2018 / Accepted: 3 July 2018 / Published online: 23 July 2018

(C) The Author(s) 2018

\begin{abstract}
Achieving a sustainable consumption and production pattern is one of the United Nation's sustainable development goals for 2030. To achieve this, it is necessary to consider the environmental burden from a product life cycle and the quality of life of the consumer. In this study, a systematic approach for connecting basic human needs and the product development process, called the living-sphere approach, is proposed. This approach is intended to encompass the complete relationship between region-specific basic needs and durable products. In this approach, value graphs, which visualize the value system of products, are connected to satisfiers fulfilling the basic needs set out by Max-Neef. A value graph links satisfiers and the traditional product development process. The significance of the proposed approach is twofold. First, improving quality of daily life and traditional product development are combined in the same framework. Second, the design of single products and the total optimization of multi-products are supported at the requirement level.
\end{abstract}

Keywords Sustainable design · Environmentally conscious design (eco-design) $\cdot$ Local community · Basic human needs · Quality of life $(\mathrm{QoL}) \cdot$ Sustainable consumption and production (SCP)

\section{Introduction}

Realizing a sustainable society is one of the most challenging problems that humanity faces. The United Nations has adopted a global framework for international cooperation, the 2030 Agenda for Sustainable Development, and the framework's Sustainable Development Goals (SDGs) [35]. In the most frequently quoted definition, sustainable development is "development that meets the needs of the present without compromising the ability of future

Hideki Kobayashi

kobayashi@mech.eng.osaka-u.ac.jp

1 Department of Mechanical Engineering, Graduate School of Engineering, Osaka University,

Yamada-oka 2-1, Suita, Osaka 565-0871, Japan 
generations to meet their own needs" [40]. Sustainable development should be seen as a process that provides humanity with a pathway to survival and living a good life on Earth [4]. In this study, we treat sustainability as an ideal system state and sustainable development as an on-going process to achieve the ideal [23].

Achieving a sustainable consumption and production (SCP) pattern [38, 39] is an SDG. Figure 1 indicates that population growth and waste generation are not decoupled in OECD countries [24]. This trend is the same as the global trend, which includes low-income countries [13]. Global waste generation in 2100 is estimated to be 2.9 times that in 2010. In the estimate [13], $72 \%$ of global waste generation in 2100 is from East Asia, Asia-Pacific, South Asia, the Middle East, North Africa, and Sub-Saharan Africa. Realizing an SCP pattern for developed countries and developing countries is critical for global sustainability.

To achieve an SCP pattern, it is necessary to consider reducing the environmental load caused by production and manufacturing and sufficiency or quality of life (QoL) of the consumer. It is expected that a package of policies, information services, and product development focusing on the improvement of QoL without increasing environmental load will be developed where high economic growth is forecast such as in Asian countries. Traditional eco-product design methods and methodologies for reducing the environmental load of a product life cycle have been developed and eco-design guidelines are being standardized $[15,37]$. Although eco-products are being diffused gradually in industrialized countries [33,34], no QoL-conscious eco-product design methodology for emergent or developing countries has been established.

In this study, locally oriented sustainable design implies that local or region-specific conditions are reflected in the specifications or eco-design ideas of a product in the design stage. We propose a systematic approach connecting the sufficiency of the daily life of a consumer and the product development process, called the living-sphere approach. We describe a framework for the living-sphere approach. We focus on the part of the function or structure that is affected strongly by region-specific conditions, such as history, culture, habits, and social infrastructure. We assume that it is impossible to separate human life completely from the land that people occupy. Related studies are surveyed in the next section, and the living-sphere approach is proposed in "Framework of the Living-sphere Approach".

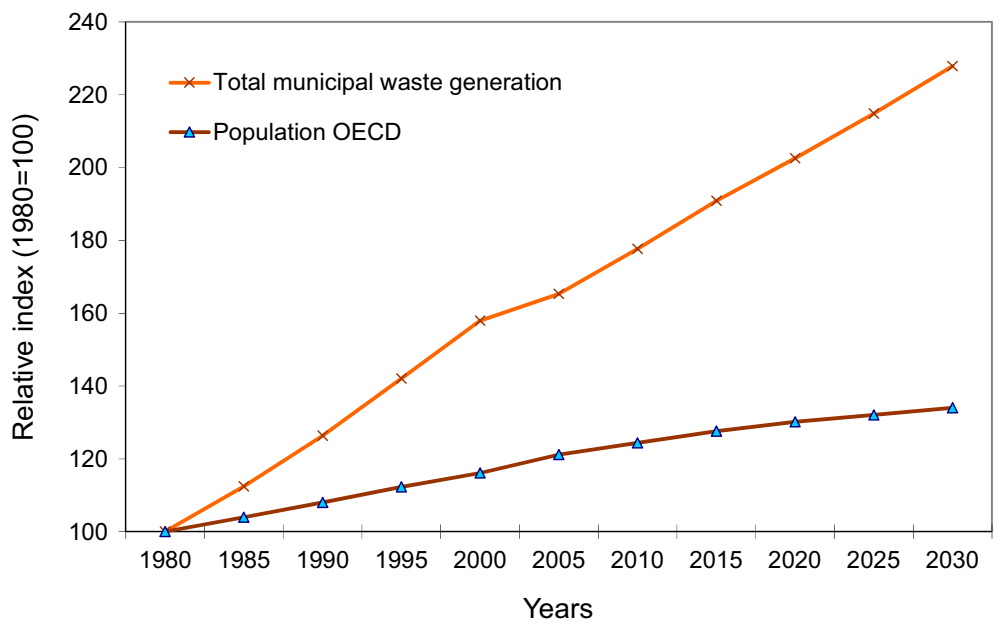

Fig. 1 Past and predicted OECD country municipal waste generation and population growth [24] 
The significance of the work and remaining issues are discussed in "Discussion", and "Concluding remarks" are provided in the final section.

\section{Related works}

The so-called factor index describes the improvement ratio of eco-efficiency, which is the product value per unit of environmental load caused by the product life cycle [19]. Although this factor index of a product can be applied to industrial products generally, the total sufficiency of the needs or the factor index of a household cannot be quantified where various products are used in daily life. A study of the factor index calculation for a household focusing on home appliances has been reported [2]. However, the method cannot consider satisfaction and region-specific characteristics because it estimates the factor index focusing on only the technological progress in the basic product functions. The concepts of eco-efficiency and sufficiency are important in SCP patterns; however, these concepts are different [7, 38]. Ecoefficiency is a management philosophy that encourages business to search for environmental improvements that yield parallel economic benefits [38]. It focuses on business opportunities to become more environmentally responsible and more profitable, therefore, it is proposed from a viewpoint of production. On the other hand, the concept of "sufficiency" has emerged over the years as an alternative economic model to consumerism and a necessary component of sustainable lifestyles. Sufficiency challenges the notion that if some is good, then more must be better; instead, it emphasizes "enoughness" [38]. This paper focuses on sufficiency rather than eco-efficiency.

Koren suggested that the personalization and regionalization in the manufacturing industry after globalization is a paradigm shift [21]. Here, we discuss personalization and regionalization in relation to the context dependency. For example, service engineering focuses on abstracting and valuing the context of the personal user and one of the methods for this is the persona approach [18]. However, it is important to abstract product specifications depending on the region-specific context through the systematic analysis of daily life [29]. It is difficult to consider the region-specific context in full via the persona approach, because this approach is insufficient in terms of relatively large spatiotemporal considerations such as the climate, culture, and institutions. Hofstede pointed out that cultural dimensions can explain many cultural differences at the region or country level [10] and there is a correlation between personality factors and cultural dimensions [11]. In this study, we focus on the differences arising from spatiotemporal factors.

Generally, the earliest development of a consumer product is a marketing process. In the traditional marketing process, the $3 \mathrm{Cs}$, namely, the customer, the competitors, and the company, are analyzed initially [26]. However, customer analysis focuses on increasing the profit of a product or service provider; thus, it does not necessarily focus on satisfying the customers' needs. In fact, it is important to observe the sufficiency of the consumers' daily needs closely. Recently, ethnography has been used as a method of field observation for product and service marketing [1]. There is no proper procedural methodology for ethnography, and the user context is understood in detail from examining daily life. The ethnographic approach is strongly affected by the subjectivity of the observer and its reproducibility is low. For business model creation, the value proposition canvas has been proposed [27], which consists of value proposition and customer segment areas. In the value proposition area, the concepts of gain creator and pain reliever are described based on the product to be supplied. 
However, gains and pains are derived based on customer jobs in the customer segment area. A product is selected if it fits with the customer's gains or pains. Because the value proposition canvas is for earning sales from segmented customers, it is not sufficient to achieve SCP for all people in the target region.

There are various study fields related to QoL studies such as human needs, welfare and well-being. Various concepts of human needs have been proposed. For instance, 30 kinds of human needs have been defined in the literature [5]. Although usually people believe that human needs change with cultural progress or period, Max-Neef claimed that basic human needs are universal, but satisfiers fulfilling the basic needs depend on region, culture, and period [22]. Here, basic human needs are the opposite of highorder needs, and they are synonymous with absolute or universal needs [5]. In welfare economics, the capability approach was proposed by Sen [28]. The capability approach focuses on freedom and opportunity of choice for measuring latent ability, and it is used for the human development index (HDI) established by the United Nations Development Programme [36]. The HDI shows an integrated value consisting of the national average lifetime, literacy rate, school attendance rate, and GDP per capita. The approach for measuring subjective well-being developed by the Organisation for Economic Cooperation and Development is a typical well-being approach [25, 30]. This approach uses a questionnaire to find out whether people are satisfied with their daily life, and it is recognized as a reliable method, although there is a lack of data for emergent or developing countries. According to the traditional perspective of the linear well-being model, a consumer accomplishes well-being based on satisfaction achieved by consuming products and services [16] (Fig. 2). Although well-being and related products are connected qualitatively in this model, there is no systematic study on the relationship between well-being and the product development process.

When we set the target product, a traditional product development methodology can be applied that focuses on required functions (What) and deploys them in the product structure (How) using a function-structure map and quality function deployment [3]. Here, in the early phase of product development, a value graph is used to confirm the reason for the existence (Why) and true value of the target product [14]. Figure 3 shows an example of a simple value graph of a hair dryer. First, the design engineer recognizes the basic values of a product by thinking why the product or requirement exists. This recognition process is used to create the upper part of the value graph. Next, the design engineer considers what functions are required for realizing the basic values and how to realize the required functions. This thought process is used to create the lower part of the value graph. Currently, this design process is used to clarify the basic values of a single

Fig. 2 Schematic of the conventional linear model [16]

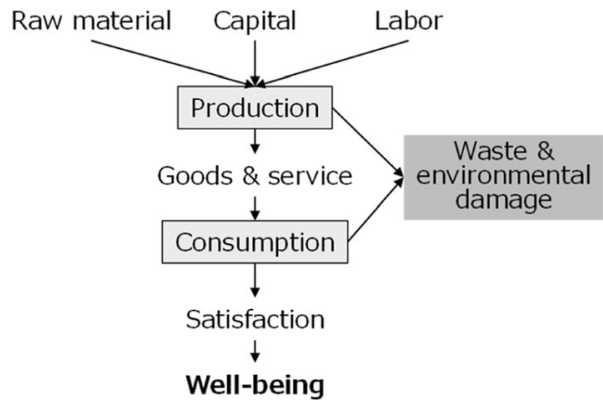




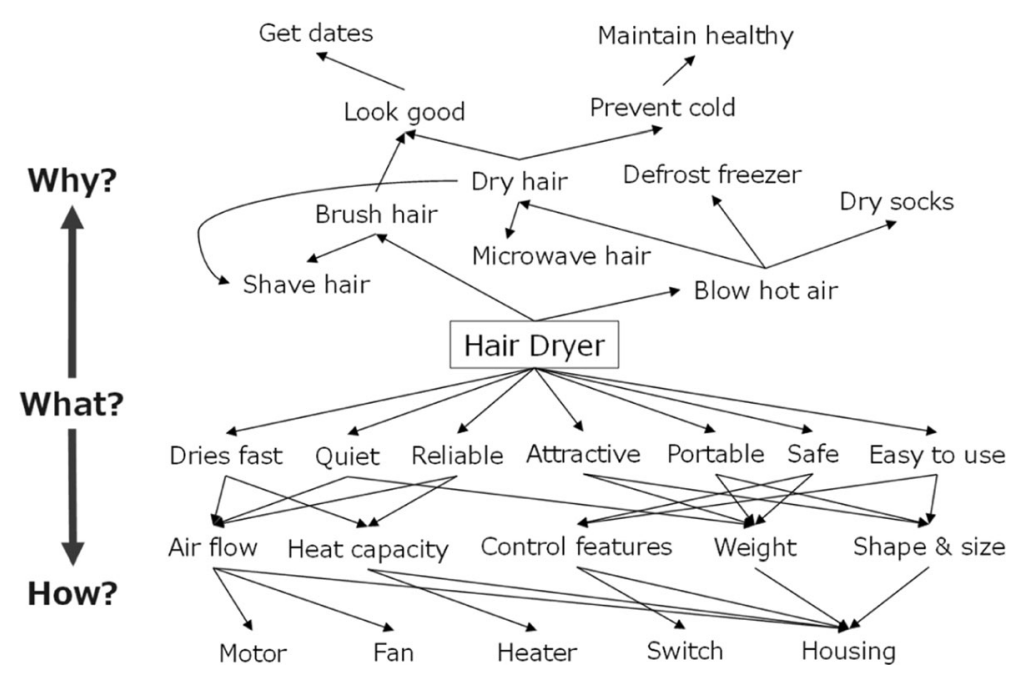

Fig. 3 Example of a value graph [14]

product. The value graph should be created by people from various departments, such as product design, research and development, marketing, sales promotion, manufacturing, and quality control, because it is useful for all these people to clarify product requirements. The basic values of a product already on the market should be re-realized when a new product is designed.

Product design support approaches for local markets, such as developing countries, include localization of an original global standardized product, sometimes called "glocalization", and co-design, which embeds local people into the product development process [20]. In glocalization, it is difficult to modify a product for a developed country to suit a developing country just by simplifying the function and structure of the product. Moreover, the design reproducibility of both approaches is low. To accomplish high reproducibility and market-in product development, we have proposed an extended function-structure map (EFSM), in which local or region-specific information is mapped on a traditional function-structure map, and a visualization system for the EFSM [20, 31].

\section{Framework of the living-sphere approach}

Figure 4 shows the framework of the living-sphere approach, and we explain the concepts used in the living-sphere approach. In the uppermost layer, human needs and satisfiers are set. Because this study is for SCP, the human needs we selected are basic needs, not egoistic infinite needs. Two categories of basic human needs proposed by Max-Neef are used [22]. As the axiological category, the following nine needs are introduced: Subsistence, Protection, Affection, Understanding, Participation, Idleness, Creation, Identity, and Freedom. False needs, which are not related to daily life, are beyond the scope of this work. As the existential category, the following four needs categories are introduced: Being, Having, Doing, and Interacting. On a matrix of the axiological and existential categories, satisfiers are set, which contribute to the actualization of human needs [22]. Satisfiers are not the available economic goods themselves 


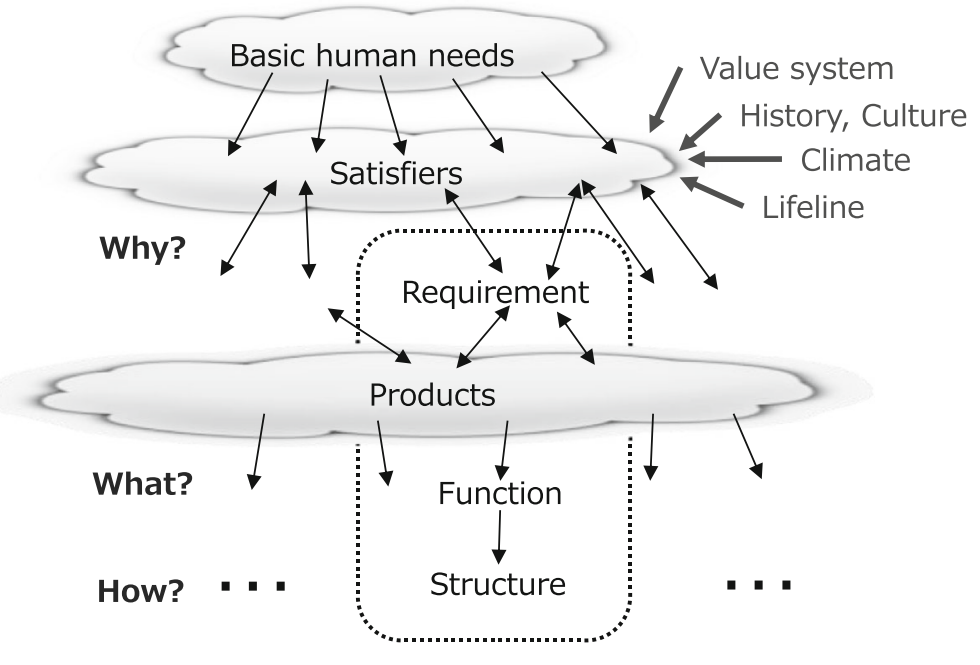

:............ Scope of value graph of a product

Fig. 4 Schematic of the living-sphere approach framework

and are selected depending on personal factors, such as religion and hobbies, and on sitespecific factors, such as regional history, culture, climate, social systems, and institutions. We focus on the site-specific factors. If the starting point of the thinking process is on the basic human needs side, a product is regarded as a method of realizing satisfiers. Thus, the value graph of the target product [14] satisfying basic needs should be connected to appropriate satisfiers.

In our framework, we assume that a design engineer can develop products satisfying basic human needs and improving QoL by confirming a connection between satisfiers and value graphs. In this way, the sufficiency of human needs and product development are connected systematically. Next, we describe the procedure for connecting related concepts during the product development process based on the proposed approach (Fig. 5).

STEP 1: The target field is investigated by a literature survey and field observations focusing on well-diffused durable products.

STEP 2: Based on the results, satisfiers realizing basic needs in the target region are abstracted and are placed in a nine-by-four categorized needs matrix by target people and analysts.

STEP 3: Value graphs of well-diffused durable products are developed by engineers, and the value graphs and satisfiers are connected.

Fig. 5 Procedure for the proposed approach

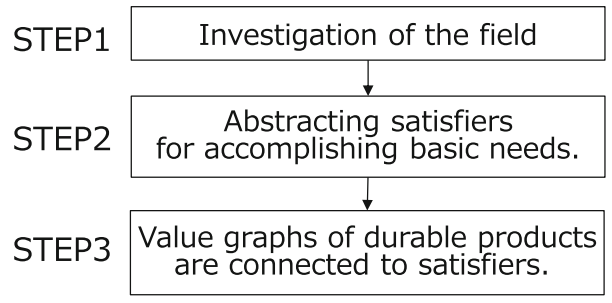


The results of these steps are interpreted by using four categories as follows (Fig. 6).

Category a): If the value graph of a product connects to one or more satisfiers, then the product is significant for satisfying basic human needs, in other words, improving QoL. In this case, region-specific information related to the whole product life cycle, namely, usage, product disposal, waste treatment and environmental load of each life cycle process is visualized on the EFSM to help improve the design [31].

Category b): If a value graph of a product does not connect to any satisfiers, then they are categorized as products that are not important for satisfying basic human needs. The priority for the product should be low in the proposed approach.

Category c): If a satisfier connects to services or actions instead of products, then it is acceptable from a viewpoint of sufficiency of basic needs.

Category d): If a satisfier does not connect to a product, service, or action, then a new concept, including a new product, service, or action, should be considered for satisfying the satisfier.

\section{Discussion}

As a simple example, the relationship between satisfiers and value graphs for refrigerators and rice cookers is shown in Fig. 7. The satisfiers abstracted by field studies are set in a matrix divided by needs categories. Black arrows represent the connections common to developing and developed countries, blue arrows represent connections for developed countries, and red arrows represent connections for developing countries. For example, the basic purpose of refrigerators is food storage; however, they are also used for drug and cosmetic storage in developed countries. These purposes are connected to the satisfiers "physical health" or "sensuality" for developed countries only. However, rice cookers in developing countries are used for cooking vegetables as well as rice. In developed countries, refrigerators and rice cookers are also connected to eating tasty food. To support eating tasty food, specific functions and parts of the products are considered. These connections are not emphasized in developing countries. In this example, two value graphs are connected to some satisfiers, allowing a design engineer to understand the relationships among the needs satisfaction, requirements, function, and structure of the two products.

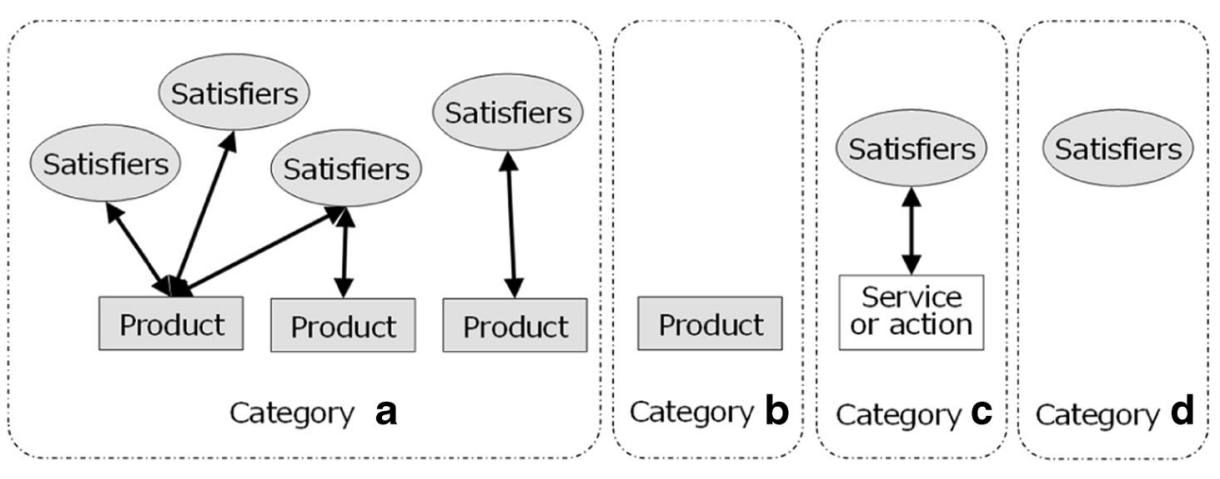

Fig. 6 Relationships between satisfiers and products 


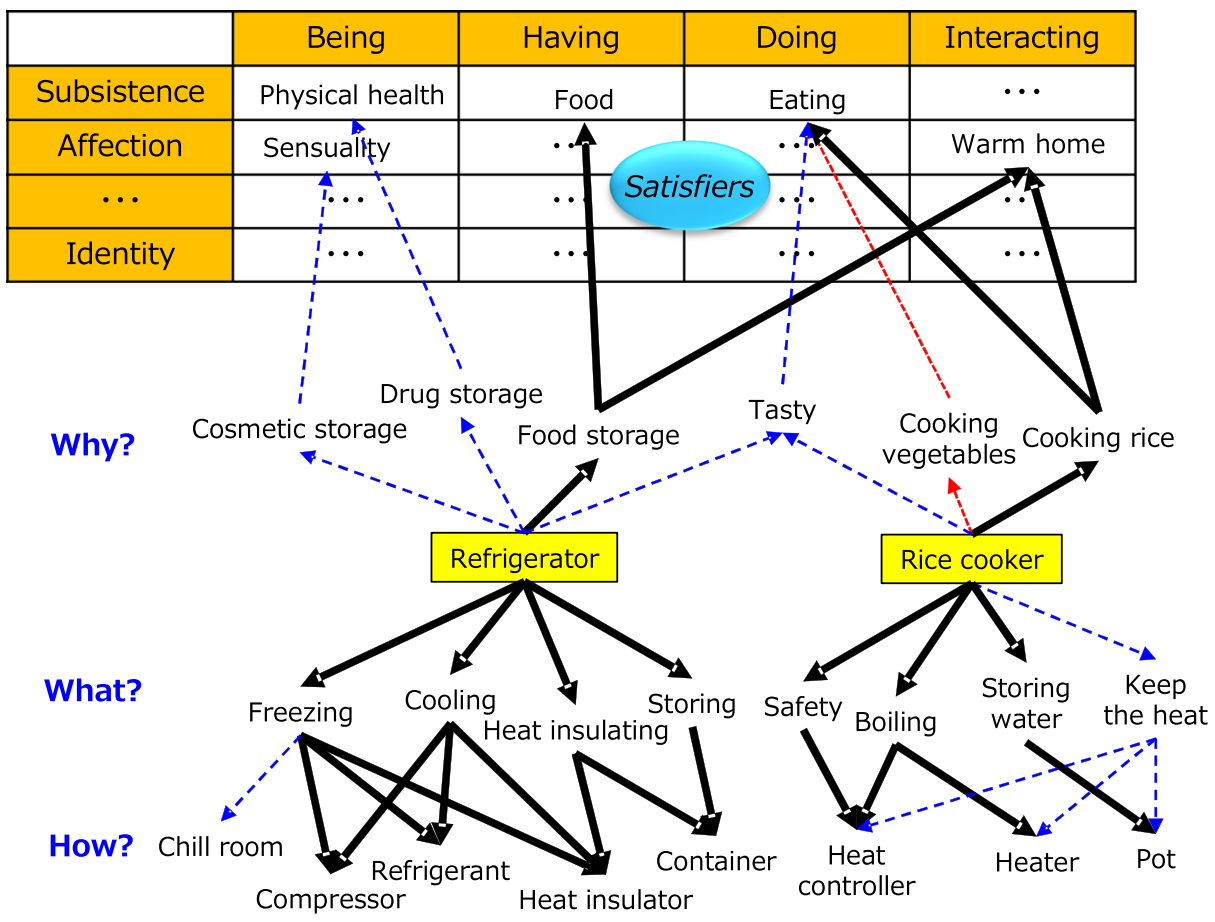

$$
\begin{aligned}
& \longrightarrow: \text { For developing \& developed countries } \\
& -\quad \text { : For developed countries } \\
& ------\rightarrow \text { For developing countries }
\end{aligned}
$$

Fig. 7 An example of satisfiers and value graphs for rice cookers and refrigerators

Figure 7 shows two overlapping diagrams for different regions. A connected diagram of satisfiers and value graphs helps to clarify the difference in product roles between regions. To improve understanding, each diagram should be drawn separately at first. In this case, there is a dependent relation among satisfiers. The "physical health" satisfier depends on "eating", and "eating" depends strictly on "food". A method that can treat this type of case will be considered in future work.

The methods in this study and the value proposition canvas [27] are similar in terms of fitting the elements deployed from the user need side and the product side. Nevertheless, these methods are clearly different from each other. As we mention in the Related Works section, the value proposition canvas is a marketing tool to increase sales; thus, it tends to focus on attractive qualities [17]. However, SCP should be achieved for all people in the target regions. This study focuses on "must-be qualities" [17], which have not been understood sufficiently, especially by design engineers in other regions. Thus, universal basic human needs are the main premise and the starting points in this study.

The importance of the proposed approach is twofold. First, the traditional single product development process is explicitly connected to improving the total sufficiency of basic needs in the same framework. Consequently, a design engineer can recognize the position of their product development project in a wider context, namely, the living sphere. Second, the total redesign or optimization of multi-products is supported at the requirement level, although it cannot be supported by existing tools, such as a value graph [14], function-structure map, and 
quality function deployment [3], for a single product. Applying the proposed approach is expected to reduce overconsumption of natural resources from selling too many kinds of product. This direction is different from the product development strategy under the mass production paradigm, namely, to manufacture many better products and release them to the market rapidly. This method for reducing overconsumption is effective for more developed countries. For consumers in developing countries, the method is expected to improve sufficiency of basic needs with minimal costs because these consumers only need to buy products obviously connected to satisfiers. The disposable income of consumers in developing countries is less than that of developed countries; thus, the proposed approach is promising in reality.

The following issues still need to be overcome.

1. Appropriate satisfiers must be set in the proposed framework. However, the selection process is not supported. Selection depends on the subjectivity of the observer and the participants. For instance, a workshop method for abstracting satisfiers has been reported [9]. One of the difficulties of handling satisfiers is that they are described in the natural language of the target region. Recognition of the external world, including nature and artefacts, depends on culture and language [6]. Although satisfiers abstracted from foreign countries must be translated when we analyse them, it is difficult in some cases. This issue is crucial in handling satisfiers; however, it should be considered in future work.

2. A support method for connecting a value graph of a product and a satisfier is needed. Here, the laddering method is used for marketing as a bottom-up method [12]. The laddering method may help to connect functions of a product and a satisfier systematically.

3. The proposed approach can be used to improve product design. However, for category d) in Fig. 6, whether a new product or service satisfies the satisfier is examined. Methods for fulfilling a satisfier are not limited to artificial and commercial goods; they depend on lifestyle, and thus on region-specific conditions. These idea generation methods require further study.

4. The proposed framework focuses on social sustainability, and is intended to fulfil the basic needs of the living sphere in the target region. A method for quantifying improvement in the sufficiency of basic needs or QoL is needed. On the other hand, balancing environmental load reduction and improvement of current QoL is essential for sustainable design. Thus, existing eco-design methods should be added to the framework.

5. Application of the proposed framework to a product-service system (PSS), including reuse and remanufacturing services, is to be considered. Recently, PSSs have focused on resource efficiency [32] and reuse [8]. This type of PSS is called a sustainable PSS. In this paper, we have focused on the connection between product requirements and the sufficiency of basic needs. However, the proposed framework can also apply to services. In future work, integration of a PSS or sustainable PSS into the proposed framework will be considered. If the values of a remanufacturing product are slightly different from those of a new product, then it can be described in the proposed framework.

\section{Concluding Remarks}

In this paper, the living-sphere approach and its framework were proposed for setting up locally oriented sustainable design. A simple example of using the proposed framework was presented. The main achievement was that the improvement of QoL and the traditional product 
development process can be connected in the proposed framework. In addition, our approach supports both designing a single product and total optimization of multi-products at the requirement level. We focused on fulfilling basic needs for achieving SCP. By recognizing the differences in satisfiers between regions, countries, or local communities, SCP can be considered more clearly in the context of climate and culture. If we introduce a PSS instead of a product in the proposed framework, then satisfiers drive a locally oriented PSS. In contrast, environmental sustainability cannot be considered explicitly in this framework because the proposed framework aims for social sustainability. Both types of sustainability should be integrated in future work. In addition, case studies will be performed for different countries to evaluate the usefulness of the proposed approach.

Acknowledgements This research was supported by JSPS KAKENHI Grant Number 15H06347, and by the Environment Research and Technology Development Fund (S-16) of the Environmental Restoration and Conservation Agency, Japan. We would like to thank Ass. Prof. Yoshinori Sumimura of Osaka University for supporting field observations.

Open Access This article is distributed under the terms of the Creative Commons Attribution 4.0 International License (http://creativecommons.org/licenses/by/4.0/), which permits unrestricted use, distribution, and reproduction in any medium, provided you give appropriate credit to the original author(s) and the source, provide a link to the Creative Commons license, and indicate if changes were made.

\section{References}

1. Arnould E, Wallendorf M (1994) Market-oriented Etnography: interpretation building and Marleting strategy formulation. J Mar Res XXXI:484-504

2. Aoe T, Yamamoto R, Ikaga T, Kondo Y, Matsuoka Y, Fukuda M (2010) Factor X (eco-efficiency) assessment on global warming a household in Japan. J Japan Inst Energy 89:1070-1087 in Japanese

3. Closing D (1993) Total quality development. ASME, New York

4. Daly H, Cobb J, Cobb C (1994) For the common good: redirecting the economy toward community, the environment, and a sustainable future. Beacon Press, Boston

5. Dean H (2010) Understanding Human Need. The Policy Press, Bristol

6. Deutscher G (2010) Through the language glass: why the world looks different in other languages. Metropolitan Books and Henry Holt and Company, New York

7. Geels F, McMeekin A, Mylan J, Southerton D (2015) A critical appraisal of sustainable consumption and production research: the reformist, revolutionary and reconfiguration positions. Glob Environ Chang 34:1-12

8. Gelbmann U, Hammerl B (2015) Integrative re-use systems as innovative business models for devising sustainable product-service-systems. J Clean Prod 97:50-60

9. Guillen-Royo M, Guardiola J, Garcia-Quero F (2017) Sustainable development in times of economic crisis: a needs-based illustration from Granada (Spain). J Clean Prod 150:267-276

10. Hofstede G (1994) Cultural dimensions in management and planning. Asia Pac J Manag:81-99

11. Hofstede G, McCrae R (2004) Personality and culture revisited: linking traits and dimensions of culture. Cross-Cult Res 38(1):52-88

12. Holtzclaw EV (2013) Laddering. Wiley, Hoboken

13. Hoornweg D, Bhada-Tata P, Kennedy C (2014) Peak waste - when is it likely to occur? J Ind Ecol 19(1):117-128

14. Ishii K (1998) Textbook of ME217 Design for Manufacture: product definition. Stanford University

15. ISO (2002) ISO/TR 14062 environmental management - integrating environmental aspects into product design and development, Geneva

16. Jackson T (2005) Motivating Sustainable Consumption. Sustainable Development Research Network, pp 10. Available via DIALOG. http://www.sustainablelifestyles.ac.uk/sites/default/files/motivating_sc_final.pdf

17. Kano N, Seraku N, Takahashi F, Tsuji S (1984) Attractive quality and must-be quality. J Japanese Soc Quality Control 14(2):39-48 in Japanese

18. Kimita K, Shimomura Y, Arai T (2009) Evaluation of customer satisfaction for PSS design. J Manuf Technol Manag 20(5):654-673 
19. Kobayashi Y, Kobayashi H, Hongu A, Sanehira K (2005) A practical method for quantifying eco-efficiency using eco-design support tools. J Ind Ecol 9(4):131-144

20. Kobayashi H (2016) Perspectives on sustainable product design methodology focused on local communities. In: Matsumoto M, Masui K, Fukushige S, Kondoh S (eds) Sustainability through innovation in product life cycle design. Springer, eBook, pp 79-92

21. Koren Y (2010) The global manufacturing revolution. John Wiley, Hoboken

22. Max-Neef M (1991) Human scale Developmenmt. The Apex Press, New York

23. Nabavi E, Daniell K, Najafi H (2017) Boundary matters: the potential of system dynamics to support sustainability? J Clean Prod 140:312-323

24. OECD (2008) Environmental Outlook to 2030

25. OECD (2013) OECD Guidelines on Measuring Sujective Well-beeing

26. Ohmae K (1991) The mind of strategist: the art of Japanese business. McGraw-Hill, New York

27. Osterwolder A, Pigneur Y, Smith A, Bernarda G, Papadakos T (2014) Value Proposition Design. Wiley, Hoboken

28. Sen A (1993) Capability and well-being. In: Nussbaum M, Sen A (eds) The quality of life. Oxford University Press, New York, pp 30-52

29. Spencer J, Lilley D, Porter S (2015) The opportunities that different cultural contexts create for sustainable design: a laundry care example. J Clean Prod 107:279-290

30. Stiglitz JE, Sen A, Fitoussi J (2010) Mismeasuring our lives: why GDP Doesn't add up. The New Press, New York

31. Sugita Y, Fukushige S, Kobayashi H (2017) A visualization system of design information for locallyoriented sustainable product. Procedia CIRP 61:617-622

32. Tukker A (2015) Product Services for a Resource-efficient and Circular Economy - a review. J Clean Prod 97:76-91

33. UK Department for Business Innovation \& Skills (2013) Low carbon environmental goods and services (LCGES), Report for 2011/2012

34. Umwelt Bundesamt (2018) Indicator: Environmentally friendly consumption, https://www. umweltbundesamt.de/en/indicator-environmentally-friendly-consumption. Accessed 25 May 2018

35. UN (2015) Sustainable Development Goals, https://www.un.org/sustainabledevelopment/sustainabledevelopment-goals/. Accessed 25 May 2018

36. UNDP (2015) Human Development Report

37. UNEP (1997) Ecodesign - a Promissing Apporach to sustainable production and consumption -, Hertfordshire

38. UNEP (2010) ABC of SCP - Clarifying Concepts on Sustainable Consumption and Production

39. UNEP (2012) Global outlook on sustainable consumption and production policies, Nairobi

40. The World Commission on Environment and Development (1987) Our Common Future 